\title{
Standardized Protocols from the Caenorhabditis Intervention Testing Program 2013-2016: Conditions and Assays used for Quantifying the Development, Fertility and Lifespan of Hermaphroditic Caenorhabditis Strains
}

Mark Lucanic ( $D$ mlucanic@buckinstitute.org)

The Buck Institute for Research on Aging, 8001 Redwood Blvd, Novato, CA 94945, USA

Monica Driscoll ( $\square$ Driscoll@dls.rutgers.edu )

Rutgers University, Dept. of Molecular Biology and Biochemistry, Nelson Biological Laboratories, Piscataway, NJ 08854, USA.

\section{Patrick Phillips}

Institute of Ecology and Evolution, University of Oregon, Eugene, OR 97403 USA

\section{Max Guo}

Division of Aging Biology, National Institute on Aging, 7201 Wisconsin Avenue, Bethesda, MD, 208929205, USA.

Jason Kish

The Buck Institute for Research on Aging, 8001 Redwood Blvd, Novato, CA 94945, USA

\section{Carolina Ibanez-Ventoso}

Rutgers University, Dept. of Molecular Biology and Biochemistry, Nelson Biological Laboratories, Piscataway, NJ 08854, USA.

\section{June Hope}

The Buck Institute for Research on Aging, 8001 Redwood Blvd, Novato, CA 94945, USA

\section{Shaunak Kamat}

Rutgers University, Dept. of Molecular Biology and Biochemistry, Nelson Biological Laboratories, Piscataway, NJ 08854, USA.

\section{Elizabeth Chao}

The Buck Institute for Research on Aging, 8001 Redwood Blvd, Novato, CA 94945, USA

\section{Shobhna Patel}

Rutgers University, Dept. of Molecular Biology and Biochemistry, Nelson Biological Laboratories, Piscataway, NJ 08854, USA.

\section{Daniel Edgar}

The Buck Institute for Research on Aging, 8001 Redwood Blvd, Novato, CA 94945, USA John Willis 
Institute of Ecology and Evolution, University of Oregon, Eugene, OR 97403 USA

\section{Mary Anne Royal}

Rutgers University, Dept. of Molecular Biology and Biochemistry, Nelson Biological Laboratories, Piscataway, NJ 08854, USA.

\section{Suzanne Angeli}

The Buck Institute for Research on Aging, 8001 Redwood Blvd, Novato, CA 94945, USA

\section{Michelle Chen}

Institute of Ecology and Evolution, University of Oregon, Eugene, OR 97403 USA

\section{Christina Chang}

Rutgers University, Dept. of Molecular Biology and Biochemistry, Nelson Biological Laboratories, Piscataway, NJ 08854, USA.

\section{Christine Sedore}

Institute of Ecology and Evolution, University of Oregon, Eugene, OR 97403 USA

\section{Manish Chamoli}

The Buck Institute for Research on Aging, 8001 Redwood Blvd, Novato, CA 94945, USA

\section{Jian Xue}

Rutgers University, Dept. of Molecular Biology and Biochemistry, Nelson Biological Laboratories, Piscataway, NJ 08854, USA.

\section{Michael Presley}

The Buck Institute for Research on Aging, 8001 Redwood Blvd, Novato, CA 94945, USA

\section{Anna Crist}

Institute of Ecology and Evolution, University of Oregon, Eugene, OR 97403 USA

\section{Suzhen Guo}

Rutgers University, Dept. of Molecular Biology and Biochemistry, Nelson Biological Laboratories, Piscataway, NJ 08854, USA.

\section{Anna Foulger}

The Buck Institute for Research on Aging, 8001 Redwood Blvd, Novato, CA 94945, USA

\section{Erik Johnson}

Institute of Ecology and Evolution, University of Oregon, Eugene, OR 97403 USA

\section{Brian Onken}

Rutgers University, Dept. of Molecular Biology and Biochemistry, Nelson Biological Laboratories, Piscataway, NJ 08854, USA.

\section{Kathleen Dumas}

The Buck Institute for Research on Aging, 8001 Redwood Blvd, Novato, CA 94945, USA

\section{Anna Coleman-Hulbert}

Institute of Ecology and Evolution, University of Oregon, Eugene, OR 97403 USA

\section{Girish Harinath}

Rutgers University, Dept. of Molecular Biology and Biochemistry, Nelson Biological Laboratories, Piscataway, NJ 08854, USA. 


\section{Dipa Bhaumik}

The Buck Institute for Research on Aging, 8001 Redwood Blvd, Novato, CA 94945, USA

\section{Esteban Chen}

Rutgers University, Dept. of Molecular Biology and Biochemistry, Nelson Biological Laboratories, Piscataway, NJ 08854, USA.

\section{Jailynn Harke}

Institute of Ecology and Evolution, University of Oregon, Eugene, OR 97403 USA

\section{W. Todd Plummer}

The Buck Institute for Research on Aging, 8001 Redwood Blvd, Novato, CA 94945, USA

\section{Gordon Lithgow}

The Buck Institute for Research on Aging, 8001 Redwood Blvd, Novato, CA 94945, USA

\section{Method Article}

Keywords: Caenorhabditis, aging, lifespan, drug discovery

Posted Date: February 7th, 2017

DOI: https://doi.org/10.1038/protex.2016.086

License: (c) (i) This work is licensed under a Creative Commons Attribution 4.0 International License. Read Full License 


\section{Abstract}

The Caenorhabditis Intervention Testing Program \(CITP) seeks to identify compounds that robustly improve lifespan and/or attenuate age-dependent declines in health across a genetically diverse set of Caenorhabditis species and strains. A core mission of the CITP is to collect data that will inform on the reproducibility of our experimental results. To achieve this, multiple replicates of each experiment are performed by each of three groups at separate laboratory sites. In an effort to limit non-biological confounding factors that may influence the reproducibility of these results, we sought to minimize differences in experimental conditions across the individual laboratories. This led to the development and utilization of a set of standardized protocols that describe specific methods for nematode maintenance and culture as well as detailed experimental procedures. All laboratories of the CITP are tasked with strict adherence to protocols. Here we provide several protocols that describe the CITP standard operating practices for bacterial cultures, media preparation and strain handing as well as assays for quantifying the development rate, fertility and lifespan of hermaphroditic Caenorhabditis strains. Individual components of these methods were not tested for their effects on outcomes and are not suggested to be best practices. However our consideration of the methodological variability available for even these simple assays does highlight the potential for differences in methodology to influence experimental reproducibility.

\section{Introduction}

Defining strategies that promote healthy aging are key priorities for maintaining human health. Although it is clear that diet, exercise, and genetics can influence longevity and health, the identification of definitive treatments that optimize healthspan and lifespan across diverse populations remains an elusive goal. Pharmacological interventions that extend lifespan or inhibit age-dependent declines in physiological function would be particularly attractive, as drugs are readily manufactured and easily administered. Individual research groups, both academic and commercial, currently deploy a variety of strategies aimed at identifying promising chemicals. The Caenorhabditis Intervention Testing Program $\backslash$ (CITP) has focused on identifying compounds that positively impact lifespan across a genetically diverse set of Caenorhabditis strains and species. This strategy is based on the hypothesis that such robust chemicals are likely to target conserved pathways and will therefore be promising drug leads for future studies in mammals. The CITP is also charged with assessing the reproducibility of its experimental results. This is accomplished in part through the requirement that all formal experiments be repeated at all three research sites. This testing of the variability of our results through experimental repeats and distributed replication was partly employed to promote confidence in our findings against growing concerns related to the reproducibility of recently published scientific literature. Specifically we refer to the concerns arising from reports that significant amounts of the recently published results from biomedical research were not reproduced when independent investigators made the attempt. This issue has triggered concern amongst the scientific community, which in turn has led funding agencies and publishers to institute new guidelines intended to promote reproducibility ${ }^{1-3}$. It is clear that reproducing results begins 
with precisely following the same technical approach and methods. In an attempt to limit the confounding effects that such methodological differences could introduce into our datasets we decided to define and strictly adhere to one set of detailed protocols amongst our distributed laboratories. Here we provide the set of detailed protocols that were followed by the CITP when collecting the data provided in our recent publication $\backslash$ (Lucanic et al in press). We also provide considerations of how these methods differ from related protocols and how these differences could influence the experimental results. _Method Development and Coordination among Labs_ To minimize experimental differences between laboratories, significant effort was devoted toward the standardization of reagents, equipment and methods and toward keeping common practices operative among groups. We first took steps to insure the same equipment and reagents were used at the three collaborating CITP institutions. We established a comprehensive ordering list for all reagents, including consumables and whenever possible coordinated orders to obtain materials with matching lot numbers. Purchasing information is noted for all supplies outlined in the Reagents and Materials List. We next developed detailed common practices and protocols that allowed the three collaborating labs to precisely replicate procedures. We accomplished this through an initial review of existing practices and experimental protocols. Positive and negative features were deliberated, taking into consideration possible influences on experimental outcome as well the general ease of implementing the method, the latter being deemed important to promote compliance from all the many researchers performing experiments that contributed to the dataset. Regularly scheduled communication between sites was used to maintain common practices and identify procedural issues that required further clarification. Representative technicians from each site held weekly meetings to compare experiences with common practices and protocols. Additional monthly phone meetings that included all team members were held to discuss issues including overall progress, protocols, data storage, quality control and reagent ordering. _Strain Selection and Distribution_ To achieve CITP goals of examining multiple Caenorhabditis strains and species with identical conditions across the distributed laboratories we obtained living organisms from a single source. These cultures were expanded and frozen down in one of the participating labs and distributed to the other sites as frozen aliquots. For the bacterial food source we used the E. coli strain OP50-1 as the Caenorhabditis food source.

_Caenorhabditis elegans_strain N2 was obtained as a working stock from the Caenorhabditis Genetics Center. That culture had been thawed from a 2007 freeze, which had been extracted from a 1995 CGC freeze. This N2 strain was used for all studies. All other worm strains were obtained from the Caenorhabditis Genetics Center or from independent researchers Asher Cutter, Marie-Anne Félix, and Christian Braendle, who provided strains that they had directly collected. Each of the CITP institutions cultured worms inside high-precision, humidity-controlled incubators for maintenance of growth conditions for CITP studies. We chose the Percival Scientific Biological Incubator model I 36NL I (temperature range $2-44^{\circ} \mathrm{C}$, set to $20 \mathrm{oC}$, humidity set to $80 \%$ relative humidity). Tolerances for each setting were $+/-0.5^{\circ} \mathrm{C}$ for temperature and $+/-10 \%$ for relative humidity.

\section{Reagents}


Reagents and Materials List Chemicals Vendor and Order Number NaCl Sigma S9888-1KG Magnesium sulfate Sigma 746452-1KG CaCl2* 2H2O Sigma 223506-1KG Cholesterol Sigma C8667-25G KH2PO4 Sigma P5379-1KG K2HP04 Sigma P3786-1KG Dimethyl Sulfoxide \(DMSO) Sigma D8418-100 ml Streptomycin Sigma S6501 50G Glycerol Calbiochem 356350-500ML Agar \(BD granulated agar) VWR\# 90000-782 2kg \(Supplier \#214510) Bacto Peptone \(BD) VWR 90000-368 2kg \(Supplier \#211820) LB Broth Miller premixed VWR 71753-5 500g FUDR VWR TCD2235 500mg Worm Culture Plates \(TC Treated) Small plates 38mm x13mm Genesee Scientific \#25-200 Medium plates 60mm x15mm Genesee Scientific \#25-260

\section{Equipment}

Percival Scientific Biological Incubator model I 36NL

\section{Procedure}

CITP Standard Operating Procedure Worm Plate Preparation General guidelines • Use CITP-standard reagents and materials as specified on the CITP Reagents and Materials List. • Record lot \# and date of opening for each reagent. • Label and dedicate CITP-only reagents. • Refer to CITP Stock Preparation chart on the last page of this section for a summary of procedures. Pre-autoclaving procedure 1 . Turn on water bath and set to $55^{\circ} \mathrm{C}$. 2. For each liter of media, add the following to a $2 \mathrm{~L}$ Erlenmeyer flask. Ingredient Amount per Liter Agar $23.0 \mathrm{~g} \mathrm{NaCl} 3.0 \mathrm{~g}$ Bacto Peptone $2.5 \mathrm{~g}$ DI Water $1 \mathrm{~L}$ Autoclave operation 1. Prepare each liter of media in a 2 liter flask. Include a large stir bar for later mixing. 2. Pour/autoclave 4 liters at once; autoclave in tray. If 4 liters is too much for one day, include "dummy" 2 liter flasks filled with 1 I mock media \( Tray: VWR 62662-241, Nalgene number 6902-5000) 3. $121^{\circ} \mathrm{C}$ at 15 psi, 45 minutes Post-autoclaving procedure 1. After autoclaving, move media immediately to $55^{\circ} \mathrm{C}$ water bath. 2. After media cools to $55^{\circ} \mathrm{C}$, add the following ingredients in the order shown below. Refer to Reagent Stock Preparations Table to prepare stocks. To Prepare 1 Liter of Media for Manual Lifespans Note: only add FUdR if appropriate for the plate type being made.

\section{Ingredient}

Potassium phosphate buffer; KP04 \ (pH 6.0)

\begin{tabular}{llll} 
Magnesium sulfate $\backslash(\mathrm{MgSO} 4)$ & $1 \mathrm{M}$ & $1 \mathrm{ml}$ & $1 \mathrm{mM}$ \\
\hline Calcium chloride $\backslash(\mathrm{CaCl} 2)$ & $1 \mathrm{M}$ & $1 \mathrm{ml}$ & $1 \mathrm{mM}$ \\
\hline Cholesterol & $5 \mathrm{mg} / \mathrm{ml}$ & $1 \mathrm{ml}$ & $5 \mathrm{mg} / \mathrm{L}$ \\
\hline FUDR $\backslash$ (if required) & $50 \mathrm{mg} / \mathrm{ml}$ & $250 \mu \mathrm{L}$ & $51 \mu \mathrm{M}$
\end{tabular}

\section{Concentration}

\section{Stock Vol. added to} Final

$25 \mathrm{ml}$

$250 \mu \mathrm{L}$
1 Liter concentration

$24 \mathrm{mM}$

Stock Vol. added to Final Ingredient Concentration 1 Liter concentration Potassium phosphate buffer $1 \mathrm{M}$

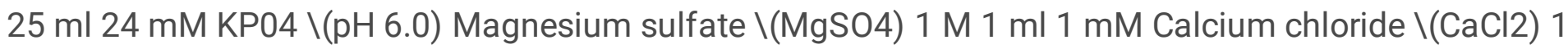


M $1 \mathrm{ml} 1 \mathrm{mM}$ Cholesterol $5 \mathrm{mg} / \mathrm{ml} 1 \mathrm{ml} 5 \mathrm{mg} / \mathrm{L}$ FUDR \(if required) $50 \mathrm{mg} / \mathrm{ml} 250 \mu \mathrm{L} 51 \mu \mathrm{M}$ Add media manually to each plate or use a calibrated plate-pouring machine. Follow the table directly below for the volume needed for the corresponding plate size. Type Media vol. per plate Used for Medium $\backslash(60 \mathrm{~mm}) 10$ $\mathrm{ml}$ strain maintenance and egg lays Small $\backslash(35 \mathrm{~mm}) 3 \mathrm{ml}$ fertility and alpha time experiments Postpouring procedure 1 . Allow plates to dry for 24 hours at room temperature or in a hood with the lids on. The next day, use a pipette to spot or "seed" the center of each plate with liquid OP50-1 suspension. Refer to CITP Standard Operating Procedure OP50-1 Bacterial Preparation and Maintenance. Refer to the table below for amount to seed based on plate size. Type of Plate Amount of OP50-1 liquid culture Medium \ $(60 \mathrm{~mm}) 200 \mu \mathrm{L}$ Small $\backslash(35 \mathrm{~mm}) 100 \mu \mathrm{L} 2$. After spotting, stack the plates 'lid up' and allow them to dry in a hood with the fan on or on a lab bench for 24 hours. Different labs have different set-ups and hood availability. The goal is to allow the lawn to dry and thicken without contamination. Then transfer plates to a tightly-covered plastic standard bin, and store 'lid up' in the CITP $20^{\circ} \mathrm{C}$ incubator for another 24 hours. 3. Flip plates to store them lid-side down at $4 \mathrm{o}$ C. Shelf Life of Plates - Under optimal conditions, plates are used up to three weeks after they are spotted. Plates treated with compounds may be used up to two weeks after treating. Continued on next page Reagent Stock Preparations Table Solute Solvent Filter Auto- Reagent Amount Solvent Volume Molarity Sterilize clave MgSO4 $2.4 \mathrm{~g}$ DI Water $20 \mathrm{~mL} 1 \mathrm{M}$ Yes Yes $\mathrm{CaCl} 22.94 \mathrm{~g}$ DI Water $20 \mathrm{~mL} 1 \mathrm{M}$ Yes No Cholesterol $100 \mathrm{mg}$ Ethanol $20 \mathrm{~mL} 5 \mathrm{mg} / \mathrm{ml}$ No No KH2PO4 $136.09 \mathrm{~g}$ DI Water $1 \mathrm{~L}$ 1M No No K2HPO4 $43.55 \mathrm{~g}$ DI Water $250 \mathrm{~mL}$ 1M No No KPO4 $200 \mathrm{ml}$ DI Water varies* 1M No Yes Monobasic FUdR $50 \mathrm{mg}$ DI Water 1mL $203 \mathrm{mM}$ Yes No *Titrate to a pH of 6.0 with dibasic buffet CITP Standard Operating Procedure OP50-1 Bacterial Preparation and Maintenance Bacterial strain: OP50-1, a streptomycin-resistant strain of E. coli OP50, is the CITP standard for bacterial lawns on worm plates. OP50-1 can be obtained from the Caenorhabditis Genetics Center. Small aliquots of OP50-1 are stored at -80D C. LB agar plates for OP50-1 bacterial growth: Prepare fresh plates each time you culture OP50-1 from frozen stock to ensure the potency of streptomycin. For each culture plate, use the following amount of reagents: Agar granules $0.75 \mathrm{~g}$ LB Broth Miller $1.25 \mathrm{~g}$ Deionized Water $50 \mathrm{~mL}$ Streptomycin $\backslash(50 \mathrm{mg} / \mathrm{mL}) 50 \mu \mathrm{L} 1$. Mix LB broth Miller granules with agar and water in a $250 \mathrm{~mL}$ flask. Autoclave and cool. Add streptomycin stock solution. Pipette $50 \mathrm{~mL}$ of media into each $10 \mathrm{~cm}$ plate. Do not use plates more than 30 days old. 2. Streak OP50-1 onto a culture plate. 3. Incubate plate at 37oC for 16 hours, store plate at $40 \mathrm{C} 4$. Streak a fresh plate every month from the frozen stock. ${ }^{\star \star \star \star N O T E: ~ T h e ~}$ CITP method for preparing plates for manual lifespan assays does not include streptomycin. Streptomycin is added to bacterial growth plates to maintain selection during the initial growth of bacterial colonies. CITP Standard Operating Procedure Nematode Maintenance General guidelines • Thaw frozen worm stocks every two months - Record the date of the thaw and relevant notes in the Data Center $\cdot$ Freshly thawed worms must be three generations out of a thaw and be free from contamination before they can be used in assays Materials needed - frozen worm stocks - 3 medium NGM plates seeded with OP50-1 per strain • P1000 pipette • sterile pipette tips \(optional: low-retention tips may reduce worm loss due to tip retention) Thawing frozen stocks 1. Remove tube $(\mathrm{s})$ containing the desired strain $\backslash(\mathrm{s})$ from $-80^{\circ} \mathrm{C}$ freezer. 2 . Thaw frozen worm tube $\backslash(\mathrm{s})$ at room temperature. Immediately pipette the liquid onto plates. a. Pipette $200 \mu \mathrm{L}$ onto each of two medium plates. b. Pipette the remaining $600 \mu \mathrm{L}$ onto the third medium plate. NOTE: Pipette the worm solution in a ring outside of the bacterial lawn in order to 
force the worms to crawl out of the solution. This helps prevent the worm solution from overwhelming the OP50-1 lawn. 3. Allow plates with lids off to dry in a hood. Store plates in the $20^{\circ} \mathrm{C}$ incubator overnight. 4 . Check the plates the next day for viable worms. Transfer $\sim 50-100$ worms to two fresh medium plates. Strain maintenance Transfer 50 eggs to a fresh medium plate $\backslash(60 \mathrm{~mm})$ twice weekly to maintain strains. Worms may be used up to two months from their thaw dates. Store the plates in covered plastic boxes in $20 \mathrm{oC}$ and $80 \%$ humidity incubator. Worms must be maintained for at least three generations after starvation, thawing, and/or contamination before being used in assays.

CITP Standard Operating Procedure Alpha Time Assay Definition: The a-time measures the "egg-to-egg time" - the interval between an egg being laid and when the emerging worm lays its first egg as an adult. Subject worms must be unmated hermaphrodites. a-Time = TFE - TELM TFE is the observed date and time of the First Egg laid by the worm; TELM is the date and time of the Midpoint of the initial Egg Lay Plan the egg lay and observation interval based on a-times observed from project strains. Materials $\backslash$ (for each strain) - egglaying worms: 50 gravid Day 1 or Day 2 unmated adult hermaphrodites of desired strain $\backslash(\mathrm{s}) \cdot 1$ medium CITP culture plate with OP50-1 bacterial lawn $\backslash$ (for egg lay) $\cdot 30$ small $\backslash(3 \mathrm{~cm})$ CITP culture plate $\backslash($ FUDR with OP50-1 lawn Procedure 1. Create the experiment record in the CITP Data Coordination Center database. Use the Alpha Time Experiment template for quick entry of metadata. 2. Set up a one-hour egg lay for each strain with approximately 50 Day 1 or Day 2 gravid adults. Keep the worms at $20^{\circ} \mathrm{C}$ for the duration of the lay. 3. Record the egg lay start time and approximate egg lay end time for each plate. Use the midpoint of that interval $\backslash(T E L M)$ for the a-time calculation. 4. After the egg lay, transfer one egg per plate onto labeled $3 \mathrm{~cm}$ plates on the bacterial lawn. The goal is to obtain 20 successful observations. 30 starting plates should yield at least 20 successful alpha-time observations. 5. Store plates in a covered box in the $20^{\circ} \mathrm{C}$ incubator until they are ready to check for alpha eggs. 6 . Once you're near the expected alpha time for the strain, check every plate once an hour. Record the time of first observed egg lay $\backslash$ (TFE) for each plate. 7. The Data Center calculates a-time using the a-time equation $\backslash(\mathrm{a}-\mathrm{Time}=\mathrm{TFE}-\mathrm{TELM})$. Note: a-time is extremely sensitive to temperature. Keep a data logger with the plates throughout worm development and scoring. Censor plates if more than five eggs have been laid at the first time of observation \(TFE). If there are more than three out of thirty plates with more than five eggs laid at the first time of observation, repeat the experiment to observe plates sooner after the initial egg lay. CITP Standard Operating Procedure Fertility Assay \(No Intervention) Materials • egg-laying worms: 50 gravid Day 1 or Day 2 adult worms, unmated hermaphrodites of desired strain $\backslash(\mathrm{s}) \cdot$ For each strain o 1 medium plate for egg lay o 20 small $\backslash(35 \times 10 \mathrm{~mm})$ seeded plates $\backslash$ (no FUdR) per days transferred $\backslash$ (usually 5-6 days) Procedure 1. Use adults for a one-hour egg lay. Burn off adults and store eggs in $20^{\circ} \mathrm{C}$. 2 . Allow the embryos on the plates to reach L4 larval stage $\backslash(\sim 48$ hours for $C$. elegans). 3. Pick individual hermaphrodite L4s for each of the 20 plates. Label each plate with the strain, date, and replicate number $\backslash$ (1-20). 4. After 24 hours, transfer the adult worm to a fresh plate. Store the plates from which you transferred the egg-laying worms for two days or until embryos reach L4. Count the number of progeny worms per plate and record that number on the data sheet or database form. 5 . Repeat steps 4 and 5 until the egg-laying cohort is no longer laying eggs. This time frame is strain-dependent, but is usually 5 to 6 days total. 6 . Record a final fertility number per subject worm by summing the number of viable progeny for all the days. CITP Standard Operating Procedure Manual Lifespan Assay General guidelines • 
Maintain a continuous culture of each strain on medium plates, kept in incubator at $20^{\circ} \mathrm{C}$ and $80 \%$ humidity $\cdot$ Use data loggers to track temperature and humidity for the duration of every assay $\cdot$ Worms must be maintained for at least three generations after starvation, thawing, and/or bacterial contamination before being used in assays - Record the egg lay date, temperature and humidity readings, strain thaw dates, etc. for each experiment - Species differences: For $\mathrm{C}$. elegans and $\mathrm{C}$. briggsae, worms reach $L 4$ stage two days after the egg lay. $C$. tropicalis reaches $L 4$ three days after the egg lay Hermaphrodite synchronization 1. Four days prior to the egg lay, transfer 60-100 eggs from each strain maintenance plate to fresh medium $\backslash(60 \mathrm{~mm})$ plates. 2 . One day prior to the egg lay, depending on timing ensure that adults are unmated. Follow "Removing Males" protocol if necessary. Removing males Spontaneous males occur at variable rates across strains and species. Rule-of-thumb: if the source plate has $>5$ males, use Method 1. If fewer, Method 2 is faster and less likely to hurt the worms. Method 1: Transfer L4 hermaphrodites from the source plate to a new seeded medium $\backslash(60 \mathrm{~mm})$ plate. Method 2: Flame off males from the source plate. Very carefully examine all the worms on the plate and flame off any males. Use remaining worms in the experiment only if visual inspection of adults reveals no adult males or signs of mating. Egg lay 1 . Transfer 50 gravid young adults $\backslash$ (day one or two of adulthood) per medium plate. The number of plates depends on how many worms you need for your experiment. 2 . Remove any eggs or larvae accidently transferred. 3. Allow worms to lay eggs for desired time at $20^{\circ} \mathrm{C} \backslash$ (usually around two hours). 4. Count the eggs laid to ensure there are more than enough for the upcoming assay. 5. NOTE: Lifespans $\backslash$ (ages) are calculated with the egg lay as Day Zero. Moving worms to assay plates 1 . Transfer 40 day-one adults to small $\backslash(35 \mathrm{~mm}) \mathrm{FUdR}+$ plates, in triplicate $\backslash($ i.e., 120 animals per strain, minimum, to start an experiment). 2. Keep plates at $20 \mathrm{oC}$ with a temperature and humidity data logger when not transferring or scoring. Transfers and scoring 1 . When transferring, move living worms to fresh small FUdR+ seeded plates warmed to room temperature. 2. Score worms according to standard CITP methods. See "Scoring recommendations" below. Schedule Friday egg lay schedule Week 1: Transfer on days $1 \backslash(\mathrm{M}), 2 \backslash(\mathrm{TU})$, and $5 \backslash(\mathrm{F})$ of adulthood. Week 2: Transfer on day $8 \backslash(\mathrm{M})$, $10 \backslash(W)$, and 12\(F) of adulthood Week 3: Transfer on day 15\(M) Week 4 and thereafter: transfer once per week $\backslash(M)$, until the worms are all dead. Score every transfer day and every Monday, Wednesday, and Friday. Monday egg lay schedule Week 1: Transfer on days $1 \backslash(\mathrm{TH})$ and $2 \backslash(\mathrm{F})$ of adulthood. Week 2 : Transfer on days $5 \backslash(\mathrm{M})$, and $7 \backslash(\mathrm{W}), 9 \backslash(\mathrm{F})$. Week 3: Transfer on days $12 \backslash(\mathrm{M})$, and $14 \backslash(\mathrm{W})$ Week 4 and thereafter: Transfer once per week $\backslash(W)$ until the worms are all dead. Score every transfer day and every Monday, Wednesday, and Friday. Continue until all worms are scored as dead or censored. Run the validation script in the database and resolve any errors. Attach the temperature and humidity spreadsheet from the data logger to the experiment record in the Filemaker database. Scoring recommendations 1. Score worms as alive, dead, lost, bagged, or extruded. 2. If worms are visibly moving, score them as alive. For static worms, assay for touch-provoked movement with a flattened platinum wire. Look for movement by prodding the tail first, then mid-body, lastly head - stopping if you see movement. 3. Record all live, dead and censored worms. Notes on scoring 1. Bagged, Lost, and Extruded are "censor" categories. "Lost" worms may be missing, burrowed or walled. 2. If bacterial contamination is observed, score all worms as lost and mark the "Censor" box for the plate in the database. Make a note of this. 3. Burrowing: Follow the " $50 \%$ rule" for plates with burrowed worms. a. If $50 \%$ or more of the worms on a plate have 
burrowed, then censor that plate on the day the burrowing is observed. b. If fewer than $50 \%$ of the worms have burrowed, then move all the worms on the surface to a fresh plate. Do not dig into the agar to extract any worms. Set the plate aside for up to an hour and check it every few minutes to see if any worms have emerged to the surface, as they frequently do. Move any accessible worms to the fresh plate. Information Sheets for Interventions and Methods for Chemical Addition to Plates Notes: To make chemical treated plates, a working solution of the chemical is prepared and added to previously seeded $\backslash$ (bacteria) and dried lifespan assay plates. After chemicals are added to plates in hoods and solutions are evenly dispersed across plates, they are allowed to dry in the hood. Immediately after drying, stack plates with lids on top and allow plates to sit in incubators $\backslash\left(20^{\circ} \mathrm{C}\right.$ and $80 \%$ humidity) for 24 hours to allow for chemical diffusion after which they can be stored at $4^{\circ} \mathrm{C}$. CITP Compound Preparation Instructions Alpha-Lipoic Acid Intervention source Company: Sigma, T1395-1g Lot \#: SLBJ6083V Container opening date: Solubility: Soluble in DMSO MW: $206.33 \mathrm{~g} / \mathrm{mol}$ Stock concentration: 40 mM Final concentration: $100 \mu \mathrm{M}$ Stock preparation and handling: To prepare $5 \mathrm{~mL}$ of stock solution in DMSO at room temperature: Add $41.27 \mathrm{mg}$ powder to $5 \mathrm{~mL}$ DMSO. Filter sterilization required? Yes Store at $-20 \mathrm{0C}$. Location: Prepare the stock solution. Sterilize with a $0.22 \mu \mathrm{m}$ filter and aliquot into sterile tubes. Plate addition. Bring $35 \mathrm{~mm}$ FUdR plates to room temperature prior to adding the compound solution. Thaw stock solution at room temperature. Mix a working solution by adding 7.5 $\mu \mathrm{L}$ of stock solution to $125 \mu \mathrm{L}$ of sterile, deionized water per plate. Spread $132.5 \mu \mathrm{L}$ of working solution evenly over each plate and allow to dry. Use notes. Label the box with the compound set and date. Store the plates lid-side down at $4^{\circ} \mathrm{C}$ for no longer than three weeks. Bring plates to room temperature before transferring worms to them. Special handling notes $\backslash$ (toxic, light sensitive?) None. Notes on anything odd about intervention plates $\backslash$ (precipitating, discolor, etc.) None. CITP Compound Preparation Instructions Alpha-Ketoglutaric Acid Intervention source Company: Sigma, K1128-5G Lot \#: BCBF0081V Container opening date: __ Powder stored at $-20^{\circ} \mathrm{C}$ Solubility: Soluble in $\mathrm{H} 2 \mathrm{O} \mathrm{MW}: 146.10 \mathrm{~g} / \mathrm{mol}$ Stock concentration: $192 \mathrm{mM}$ Final concentration: $8.0 \mathrm{mM}$ Stock preparation and handling: To prepare the 192 $\mathrm{mM}$ stock in $\mathrm{DI} \mathrm{H} 2 \mathrm{O}$ at room temperature, add $0.70 \mathrm{~g}$ powdered compound to $25 \mathrm{~mL}$ DI H2O. Invert the mixing tube to dissolve. Sterilize with $0.22 \mu \mathrm{m}$ filters into a sterile tube. As a general guideline, $12.5 \mathrm{~mL}$ will treat approximately 90 small $\backslash(35 \mathrm{~mm})$ plates. Filter sterilization required? Yes Store at $-200 \mathrm{C}$. Location: Aliquot stock into sterile tubes. Unless using immediately, freeze tubes for storage at $-20^{\circ} \mathrm{C}$. Plate addition Bring $35 \mathrm{~mm}$ FUdR plates to room temperature. Pipette $125 \mu \mathrm{L}$ of stock solution to each plate. Gently swirl plates to spread compound evenly. Use notes. Label the box with the compound set and date. Store the plates lid-side down at $4^{\circ} \mathrm{C}$ for no longer than three weeks. Bring plates to room temperature before transferring worms to them. Special handling notes $\backslash$ (toxic, light sensitive?) None. Notes on anything odd about intervention plates $\backslash$ (precipitating, discolor, etc.) None. CITP Compound Preparation Instructions Aspirin Intervention source Company: Sigma, A2093-100g Lot \#: MKBQ8444V Container opening date: Solubility: Soluble in DMSO MW: $180.16 \mathrm{~g} / \mathrm{mol}$ Stock concentration: $40 \mathrm{mM}$ Final concentration: $100 \mu \mathrm{M}$ Stock preparation and handling: To prepare $5 \mathrm{~mL}$ of stock solution in DMSO at room temperature: Add $36.03 \mathrm{mg}$ powder to $5 \mathrm{~mL}$ DMSO. Filter sterilization required? Yes Store at -200 C. Location: Prepare the stock solution. Sterilize with a $0.22 \mu \mathrm{m}$ filter and aliquot into sterile tubes. Plate addition. Bring $35 \mathrm{~mm}$ FUdR plates to room 
temperature prior to adding the compound solution. Thaw stock solution at room temperature. Mix a working solution by adding $7.5 \mu \mathrm{L}$ of stock solution to $125 \mu \mathrm{L}$ of sterile, deionized water per plate. Spread $132.5 \mu \mathrm{L}$ of working solution evenly over each plate and allow to dry. Use notes. Label the box with the compound set and date. Store the plates lid-side down at $4^{\circ} \mathrm{C}$ for no longer than three weeks. Bring plates to room temperature before transferring worms to them. Special handling notes $\backslash$ (toxic, light sensitive?) None. Notes on anything odd about intervention plates $\backslash$ (precipitating, discolor, etc.) None. CITP Compound Preparation Instructions Curcumin Intervention source Company: Sigma Lot \#: MKBR0090V Container opening date: Solubility: Soluble in DMSO MW: $368.38 \mathrm{~g} / \mathrm{mol}$ Stock concentration: $40 \mathrm{mM}$ Final concentration: $100 \mu \mathrm{M}$ Stock preparation and handling: To prepare $5 \mathrm{~mL}$ of stock solution at room temperature: Add $73.68 \mathrm{mg}$ powder to $5 \mathrm{~mL}$ DMSO. Filter sterilization required? Yes Store at $-20 \mathrm{0C}$. Location: Prepare the stock solution. Sterilize with a $0.22 \mu \mathrm{m}$ PVDF filter and aliquot into tubes. Plate addition Bring $35 \mathrm{~mm}$ FUdR plates to room temperature prior to adding the compound solution. Completely thaw the stock solution at room temperature. Compound precipitates at concentration of working solution so individual plate aliquots are prepared as the working solution. Prepare individual aliquots of $7.5 \mu \mathrm{L}$ of stock solution in small 'PCR' tubes. Add $125 \mu \mathrm{L}$ of sterile, deionized water. Pipet the entirety of this onto the assay plate, evenly distribute across the plate and allow to dry. Use notes. Label the box with the compound set and date. Store the plates lid-side down at $4^{\circ} \mathrm{C}$ for no longer than three weeks. Bring plates to room temperature before transferring worms to them. Special handling notes $\backslash$ (toxic, light sensitive?) None Notes on anything odd about intervention plates $\backslash$ (precipitating, discolor, etc.) Precipitates at final concentration. CITP Compound Preparation Instructions NP1 Intervention source Company: Chembridge Labs Lot \#: N/A. Custom order; supply distributed by Lithgow lab Container opening date: Click here to enter text. Solubility: Soluble in DMSO MW: $336 \mathrm{~g} / \mathrm{mol}$ Stock concentration: $20 \mathrm{mM}$ Final concentration: $50 \mu \mathrm{M}$ Stock preparation and handling: To prepare 20 $\mathrm{mM}$ stock in DMSO at room temperature: Add $33.6 \mathrm{mg}$ powder to $5 \mathrm{~mL}$ DMSO. Filter sterilization required? Yes Store at $-20 \mathrm{OC}$. Location: Prepare the stock solution. Sterilize with a $0.22 \mu \mathrm{m}$ PVDF filter and aliquot into tubes. Plate addition Bring $35 \mathrm{~mm}$ FUdR plates to room temperature prior to adding the compound solution. Completely thaw the stock solution at room temperature. Compound precipitates at concentration of working solution so individual plate aliquots are prepared as the working solution. Prepare individual aliquots of $7.5 \mu \mathrm{L}$ of stock solution in small 'PCR' tubes. Add $125 \mu \mathrm{L}$ of sterile, deionized water. Pipet the entirety of this onto the assay plate, evenly distribute across the plate and allow to dry. Use notes. Label the box with the compound set and date. Store the plates lid-side down at $4^{\circ} \mathrm{C}$ for no longer than three weeks. Bring plates to room temperature before transferring worms to them. Special handling notes \(toxic, light sensitive?) None Notes on anything odd about intervention plates $\backslash$ (precipitating, discolor, etc.) Precipitates at working solution concentration CITP Compound Preparation Instructions Propyl Gallate Intervention source Company: Sigma, P3130-100g Lot \#: MKBR8169V Container opening date: Solubility: Soluble in DMSO MW: 212.20 g/mol Stock concentration: $80 \mathrm{mM}$ Final concentration: $200 \mu \mathrm{M}$ Stock preparation and handling: To prepare $5 \mathrm{~mL}$ of stock solution in DMSO at room temperature: Add $84.88 \mathrm{mg}$ powder to $5 \mathrm{~mL}$ DMSO. Filter sterilization required? Yes Store at $-200 \mathrm{C}$. Location: Prepare the stock solution. Sterilize with a $0.22 \mu \mathrm{m}$ filter and aliquot into sterile tubes. Plate addition. Bring $35 \mathrm{~mm}$ FUdR 
plates to room temperature prior to adding the compound solution. Thaw stock solution at room temperature. Mix a working solution by adding $7.5 \mu \mathrm{L}$ of stock solution to $125 \mu \mathrm{L}$ of sterile, deionized water per plate. Spread $132.5 \mu \mathrm{L}$ of working solution evenly over each plate and allow to dry. Use notes. Label the box with the compound set and date. Store the plates lid-side down at $4^{\circ} \mathrm{C}$ for no longer than three weeks. Bring plates to room temperature before transferring worms to them. Special handling notes $\backslash$ (toxic, light sensitive?) None. Notes on anything odd about intervention plates $\backslash$ (precipitating, discolor, etc.) None. CITP Compound Preparation Instructions Quercetin Intervention source Company: Sigma, Q4951-10g Lot \#: SLBK4625V Container opening date: Solubility: Soluble in DMSO MW: $302.24 \mathrm{~g} / \mathrm{mol}$ Stock concentration: $40 \mathrm{mM}$ Final concentration: $100 \mu \mathrm{M}$ Stock preparation and handling: To prepare 5 $\mathrm{mL}$ of stock solution in DMSO at room temperature: Add $60.45 \mathrm{mg}$ powder to $5 \mathrm{~mL}$ DMSO. Filter sterilization required? Yes Store at $-20 \mathrm{OC}$. Location: Prepare the stock solution. Sterilize with a $0.22 \mu \mathrm{m}$ PVDF filter and aliquot into tubes. Plate addition Bring $35 \mathrm{~mm}$ FUdR plates to room temperature prior to adding the compound solution. Completely thaw the stock solution at room temperature. Compound precipitates at concentration of working solution so individual plate aliquots are prepared as the working solution. Prepare individual aliquots of $7.5 \mu \mathrm{L}$ of stock solution in small 'PCR' tubes. Add $125 \mu \mathrm{L}$ of sterile, deionized water. Pipet the entirety of this onto the assay plate, evenly distribute across the plate and allow to dry. Use notes. Label the box with the compound set and date. Store the plates lid-side down at $4^{\circ} \mathrm{C}$ for no longer than three weeks. Bring plates to room temperature before transferring worms to them. Special handling notes $\backslash$ (toxic, light sensitive?) None Notes on anything odd about intervention plates $\backslash$ (precipitating, discolor, etc.) Precipitates at final concentration. CITP Compound Preparation Instructions Resveratrol Intervention source Company: Cayman Chemical Company Lot \#: 0414330-182 Container opening date: Solubility: Soluble in DMSO MW: $228.24 \mathrm{~g} / \mathrm{mol}$ Stock concentration: $40 \mathrm{mM}$ Final concentration: $100 \mu \mathrm{M}$ Stock preparation and handling: To prepare $5 \mathrm{~mL}$ of stock solution in DMSO at room temperature: Add $45.65 \mathrm{mg}$ powder to 5 $\mathrm{mL}$ DMSO. Filter sterilization required? Yes Store at $-20 \mathrm{0C}$. Location: Prepare the stock solution. Sterilize with a $0.22 \mu \mathrm{m}$ filter and aliquot into sterile tubes. Plate addition. Bring $35 \mathrm{~mm}$ FUdR plates to room temperature prior to adding the compound solution. Thaw stock solution at room temperature. Mix a working solution by adding $7.5 \mu \mathrm{L}$ of stock solution to $125 \mu \mathrm{L}$ of sterile, deionized water per plate. Spread $132.5 \mu \mathrm{L}$ of working solution evenly over each plate and allow to dry. Use notes. Label the box with the compound set and date. Store the plates lid-side down at $4^{\circ} \mathrm{C}$ for no longer than three weeks. Bring plates to room temperature before transferring worms to them. Special handling notes $\backslash$ (toxic, light sensitive?) None. Notes on anything odd about intervention plates $\backslash$ (precipitating, discolor, etc.) None. CITP Compound Preparation Instructions Thioflavin T Intervention source Company: MP Biomedicals Lot \#: M6490 Container opening date: Solubility: Soluble in H2O MW: $318.86 \mathrm{~g} / \mathrm{mol}$ Stock concentration: $1.2 \mathrm{mM}$ Final concentration: $50 \mu \mathrm{M}$ Stock preparation and handling: To prepare $1.2 \mathrm{mM}$ stock in DI H2O at room temperature, make at least $50 \mathrm{~mL}$ of stock solution since the amount of powdered drug is so small. To make $50 \mathrm{~mL}$ of solution, add $19.1 \mathrm{mg}$ powdered drug to $50 \mathrm{~mL}$ water. Filter-sterilize with a $0.22 \mu \mathrm{m}$ filter and aliquot into sterile $1.5 \mathrm{~mL}$ tubes. Filter sterilization required? Yes Store at -20 0C. Location: Aliquot stock into sterile tubes. Unless using immediately, freeze tubes for storage at $-20^{\circ} \mathrm{C}$. Plate addition Bring $35 \mathrm{~mm}$ FUdR plates to room 
temperature. Pipette $125 \mu \mathrm{L}$ of stock solution to each plate. Gently swirl plates to spread compound evenly. Use notes. Label the box with the compound set and date. Store the plates lid-side down at $4^{\circ} \mathrm{C}$ for no longer than three weeks. Bring plates to room temperature before transferring worms to them. Special handling notes $\backslash$ (toxic, light sensitive?) ThioT is light sensitive. Keep plates on bench for as short as time as possible. Notes on anything odd about intervention plates $\backslash$ (precipitating, discolor, etc.) None. CITP Compound Preparation Instructions Valproic Acid Intervention source Company: Sigma Lot \#: MKBS5723V Container opening date: Solubility: Soluble in H2O MW: $166.19 \mathrm{~g} / \mathrm{mol}$ Stock concentration: $72 \mathrm{mM}$ Final concentration: $3 \mathrm{mM}$ Stock preparation and handling: To prepare $7.2 \mathrm{mM}$ stock solution in deionized water at room temperature: Add $0.30 \mathrm{~g}$ powder to $25 \mathrm{~mL}$ water. Sterilize using $0.22 \mathrm{um}$ filter and aliquot into sterile tubes. Filter sterilization required? Yes Store at -20 0C. Location: Aliquot stock into sterile tubes. Unless using immediately, freeze tubes for storage at $-20^{\circ} \mathrm{C}$. Plate addition Bring $35 \mathrm{~mm}$ FUdR plates to room temperature. Pipette $125 \mu \mathrm{L}$ of stock solution to each plate. Gently swirl plates to spread compound evenly. Use notes. Label the box with the compound set and date. Store the plates lid-side down at $4^{\circ} \mathrm{C}$ for no longer than three weeks. Bring plates to room temperature before transferring worms to them. Special handling notes $\backslash$ (toxic, light sensitive?) None. Notes on anything odd about intervention plates $\backslash$ (precipitating, discolor, etc.) None.

\section{Anticipated Results}

Discussion This methods report was prepared to share the detailed protocols used by the CITP during the set of experiments described in our first publication \(Lucanic et al in press). Our goals for that research was to define a set of protocols that could be strictly followed by the many researchers performing those experiment in an effort to reduce low reproducibility across the three sites arising from methodological differences. We did not directly test whether differences in methodology would significantly alter outcomes. The methods presented here, particularly related to the lifespan assays, are common methods used in the_C. elegans_aging field. However they are by no means completely inclusive of all the distinct conditions used in the field as a whole. Instead our protocols contain just one set of the possible conditional variables that could affect experimental outcomes. In particular we used live bacteria, used the sterility inducing compound FUdR and added test chemicals directly to assay plates. Variations in any, or all of these methods could reasonably be expected to result in different experimental outcomes. Specifically, live bacteria have been shown to influence chemical lifespan assays 4,5 . Live cultures are metabolically active and could therefore alter $\backslash$ (causing a positive or negative outcome) the chemicals being tested in the lifespan assay. Additionally the chemical being tested would also be available to act on the bacterial food source leaving open the possibility that any effect on the nematode lifespan under study could be an indirect effect from chemical action on the bacteria. All of our lifespan assays contained $51 \mu \mathrm{m}$ FUdR in plates, a low-level dose that has been shown to not impact the lifespan of the N2 strain under standard growth conditions 6 . However the effects of the same levels have not been reported for the other 21 nematode strains we used in our study. That this concentration of FUdR could influence these other strains lifespan remains a possibility. Further, and it has previously been reported that FUdR alters the lifespan of specific genetic mutants in an otherwise N2 background and can effect 
phenotypes other than fertility 7-9. Adding chemicals directly to lifespan assay plates could also alter experimental outcomes as it required an initial high dose of chemical exposure to the live bacteria culture and requires diffusion of chemical throughout culture media. The results of our lifespan experiments may have been influenced in an unknown way by these specific methods and further tests will be required to determine what, if any effects these specific methods had on the outcomes. Despite these caveats we suggest that these methods can act as a guide for future studies testing the development, fertility and lifespan of hermaphroditic Caenorhabditis species and strains.

\section{References}

1 Baker, M. 1,500 scientists lift the lid on reproducibility. Nature 533, 452-454, doi:10.1038/533452a \ (2016). 2 Collins, F. S. \& Tabak, L. A. Policy: NIH plans to enhance reproducibility. Nature 505, 612-613। (2014). 3 Freedman, L. P., Cockburn, I. M. \& Simcoe, T. S. The Economics of Reproducibility in Preclinical Research. PLoS biology 13, e1002165, doi:10.1371/journal.pbio.1002165 \(2015). 4 Cabreiro, F. et al. Metformin retards aging in C. elegans by altering microbial folate and methionine metabolism. Cell 153, 228-239, doi:10.1016/j.cell.2013.02.035 \(2013). 5 Onken, B. \& Driscoll, M. Metformin induces a dietary restriction-like state and the oxidative stress response to extend C. elegans Healthspan via AMPK, LKB1, and SKN-1. PLoS ONE 5, e8758, doi:10.1371/journal.pone.0008758 \(2010). 6 Van Raamsdonk, J. M. \& Hekimi, S. FUdR causes a twofold increase in the lifespan of the mitochondrial mutant gas-1. Mechanisms of ageing and development 132, 519-521, doi:10.1016/j.mad.2011.08.006 \(2011). 7 Aitlhadj, L. \& Sturzenbaum, S. R. The use of FUdR can cause prolonged longevity in mutant nematodes. Mechanisms of ageing and development 131, 364-365, doi:S0047-6374\(10)00050-3 \[pii] 10.1016/j.mad.2010.03.002 \(2010). 8 Angeli, S. et al. A DNA synthesis inhibitor is protective against proteotoxic stressors via modulation of fertility pathways in Caenorhabditis elegans. Aging \(Albany NY) 5, 759-769 \(2013). 9 Feldman, N., Kosolapov, L. \& Ben-Zvi, A. Fluorodeoxyuridine improves Caenorhabditis elegans proteostasis independent of reproduction onset. PLoS ONE 9, e85964, doi:10.1371/journal.pone.0085964 \(2014).

\section{Acknowledgements}

We acknowledge members of the Lithgow, Driscoll and Phillips labs for helpful discussions particularly focused on detailed protocols. We also thank the CITP Advisory Committee and Ronald Kohanski \ (National Institute on Aging). We thank Asher Cutter, Marie-Anne Félix, and Christian Braendle for providing strains that they had directly collected. Additional strains were provided by the CGC, which is funded by NIH Office of Research Infrastructure Programs \(P40 OD010440). This work was supported by funding from the Larry L. Hillblom Foundation, the Glenn Foundation for Medical Research and NIH grants \(UL1024917, supporting the Interdisciplinary Research Consortium on Geroscience, 1R01AG029631, R21AG048528, U01AG045844, U01AG045864). 